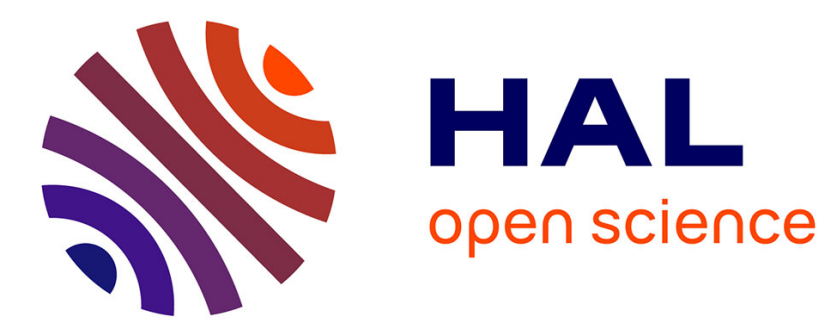

\title{
Periodic trajectories in Aeolian sand transport
}

Alexandre Valance, J.T. Jenkins

\section{To cite this version:}

Alexandre Valance, J.T. Jenkins. Periodic trajectories in Aeolian sand transport. Physics of Fluids, 2014, 26 (7), pp.073301. 10.1063/1.4885576 . hal-01020442

\section{HAL Id: hal-01020442 https://hal.science/hal-01020442}

Submitted on 8 Jul 2014

HAL is a multi-disciplinary open access archive for the deposit and dissemination of scientific research documents, whether they are published or not. The documents may come from teaching and research institutions in France or abroad, or from public or private research centers.
L'archive ouverte pluridisciplinaire HAL, est destinée au dépôt et à la diffusion de documents scientifiques de niveau recherche, publiés ou non, émanant des établissements d'enseignement et de recherche français ou étrangers, des laboratoires publics ou privés. 


\title{
Periodic trajectories in Aeolian sand transport
}

\author{
J. T. Jenkins ${ }^{1}$ and A. Valance ${ }^{2}$ \\ ${ }^{1}$ School of Civil and Environmental Engineering, Cornell University, Ithaca, NY 14853, USA \\ 2 Institut de Physique de Rennes, UMR CNRS 6251, Université de Rennes1, 35042 Rennes, France
}

(Dated: 07/07/2014)

\begin{abstract}
We develop a simple model for steady, uniform transport in aeolian saltation over a horizontal bed that is based on the computation of periodic particle trajectories in a turbulent shearing flow. The wind and the particles interact through drag, and the particles collide with the bed. We consider collisions with both rigid and erodible beds. The impact velocity in a periodic trajectory over a rigid bed is unconstrained, while that over an erodible bed must have a value that produces a single rebounding particle. The difference in the nature of the collisions results in qualitative differences in the nature of the solutions for the periodic trajectories and, in particular, to differences in the dependence of the particle flow rate on the strength of the turbulent shearing.
\end{abstract}

\section{INTRODUCTION}

When a turbulent wind blowing over bed of sand becomes sufficiently strong, a grain may be lifted from the bed by a strong, localized turbulent eddy. The drag of the air then accelerates it, and it collides with the bed with increased momentum. Impacting grains rebound and eject other grains that may also be accelerated by the wind until a sufficient number of grains are participating in the process to diminish the wind near the bed and create a steady balance in the exchanges of momentum between the grains and the wind and the grains and the bed. The result is a steady cloud of grains that jump (or saltate) over the bed. This saltation is the primary mode of sand movement [1].

Subsequently, sand may be transported by the turbulent wind over a rigid, bumpy bed. In a steady, uniform flow of this type, the horizontal flux of particles is maintained and the profiles of the wind velocity and the particle concentration and average velocity are determined by the particles' interaction with the bed. The horizontal particle flux may range in value from zero to a maximum that depends on the strength of the wind.

Discrete numerical simulations, e.g., [2-7], have the capacity to reproduce the profiles of the average particle and wind velocities and particle concentration measured in the laboratory and in the field. The most recent of these simulations [5-7] capture the interactions between individual particles and the wind and the collisions between the particles, both above and within the bed. They can provide the basis for and the means of testing scaling relations in steady flows [6].

Such simulations are detailed and relative complete. Here, we introduce a far simpler simulation that seems to capture the essential feature of steady flows over rigid and erodible beds. We focus on the upward and downward trajectories of a single particle that undergoes periodic motion subject to the drag of a steady, uniform turbulent shearing flow. We take the trajectory to be the average of the trajectories that participate in a steady motion. Individual trajectories will differ in phase and exhibit a range of heights and lengths. Models for saltation over erodible beds $[8,9]$ sometimes distinguish between low- and high-energy particles. Because we are interested in the average properties of steady flows, rather than, for example, ripple formation, we are content to predict the features of the single, periodic trajectory.

We link the downward and upward velocities at the bed by a function that provides the relationship between the velocities of the incoming and outgoing particles. We consider impacts both with a rigid, bumpy bed [10-12] and with an erodible particle bed [13-21].

The numerical determination of the resulting periodic trajectories includes their height and length and permits the calculation of the profiles of average wind and particle velocities and particle concentration. The relations between the total particle flux and the strength of the turbulent shearing flow determined from the profiles are different for the two types of bed and agree with the forms obtained in more detailed numerical simulation and physical experiments. The periodic trajectories are simple and relatively easy to analyze. From them, we determine analytical relationship between the features of the particle transport over both types beds. These confirm, extend, and make more precise existing scalings (e.g., [6]).

\section{MODEL EQUATIONS}

We provide a simple description of saltating particles within a turbulent boundary layer that interact with horizontal beds of two different types: rigid, bumpy; and erodible. 


\section{A. Particle motion}

We consider particles of mass $m$ made of a material with a mass density $\rho^{s}$ that interact with gravity and a shear flow of a turbulent gas with mass density $\rho^{f}$ and viscosity $\mu^{f}$. The gravitational acceleration is $g$, the average horizontal velocity of the wind is $U$, and the horizontal and vertical components of the particle velocity are $\xi_{x}$ and $\xi_{y}$. The flow is steady and uniform, so the velocities are functions only of the upward vertical coordinate $y$. A sketch of the situation is shown in Fig. 1.

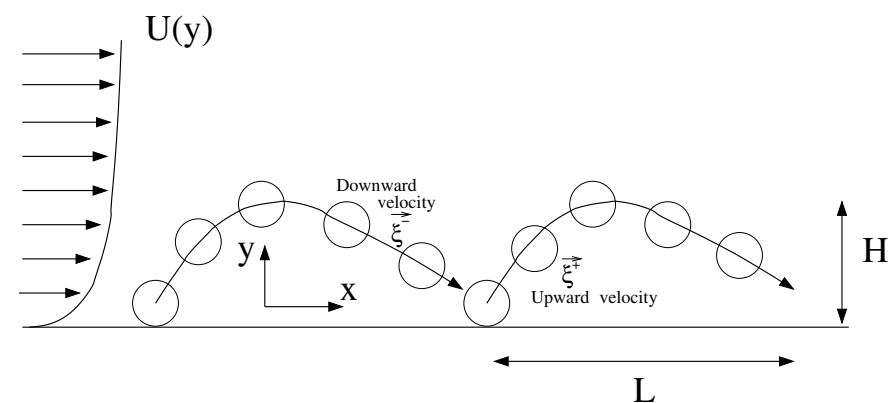

FIG. 1: A sketch of saltation.

Lengths are made dimensionless by the diameter $d$ of the particle, velocities are made dimensionless by $(g d)^{1 / 2}$, and stresses are made dimensionless by $\rho^{s} g d$. The dimensionless, nonlinear drag coefficient is denoted by $D$ and has the form [22]:

$$
D=\left(0.3 \sqrt{\left(U-\xi_{x}\right)^{2}+\xi_{y}^{2}}+18 / R\right) / \sigma
$$

where $\sigma=\rho^{s} / \rho^{f}$ and $R=\rho^{f} d(g d)^{1 / 2} / \mu^{f}$.

In the following, we will use plus and minus superscripts to label, respectively, quantities in the upward and downward parts of a trajectory. The upward and downward components of the particle velocity are governed by

$$
\begin{aligned}
& \xi_{y}^{+} \frac{d \xi_{x}^{+}}{d y}=D^{+}\left(U-\xi_{x}^{+}\right), \\
& \xi_{y}^{+} \frac{d \xi_{y}^{+}}{d y}=-D^{+} \xi_{y}^{+}-1,
\end{aligned}
$$

and

$$
\begin{aligned}
& \xi_{y}^{-} \frac{d \xi_{x}^{-}}{d y}=D^{-}\left(U-\xi_{x}^{-}\right) \\
& \xi_{y}^{-} \frac{d \xi_{y}^{-}}{d y}=-D^{-} \xi_{y}^{-}-1
\end{aligned}
$$

respectively.

The horizontal space variables $x^{+}$and $x^{-}$can be considered as functions of $y$ and obey the following first order equations:

$$
\xi_{y}^{+} \frac{d x^{+}}{d y}=\xi_{x}^{+}
$$

and

$$
\xi_{y}^{-} \frac{d x^{-}}{d y}=\xi_{x}^{-}
$$

The definitions of the average particle velocity $u$, and the particle shear stress $s$ are

$$
u \equiv\left(c^{+} \xi_{x}^{+}+c^{-} \xi_{x}^{-}\right) /\left(c^{+}+c^{-}\right),
$$


and

$$
s \equiv-\left(c^{+} \xi_{y}^{+} \xi_{x}^{+}+c^{-} \xi_{y}^{-} \xi_{x}^{-}\right)
$$

where $c^{+}$and $c^{-}$are, respectively, the concentration of ascending and descending particles. In steady, uniform saltation, the vertical fluxes of ascending and descending particles, $\phi^{+}$and $\phi^{-}$, balance exactly at any point, so

$$
\phi^{+}=-\phi^{-}=c^{+} \xi_{y}^{+}=-c^{-} \xi_{y}^{-} .
$$

Furthermore, by virtue of mass conservation, the vertical fluxes are independent of the vertical position in a steady and uniform flow. For any point $y \leq H$, where $H$ is the height of the trajectory, we thus have:

$$
\phi^{+}(y)=-\phi^{-}(y)=\phi^{+}(0) \equiv \phi_{0}
$$

\section{B. Air flow}

To solve for the air flow, we use the fact that the sum of the particle and gas shear stresses (respectively, $s$ and $S$ ) is constant through the flow,

$$
s+S=S^{*},
$$

where $S^{*}$, the Shields parameter, is the dimensionless shear stress in the particle-free gas. The relation between the shear rate and the shear stress of the wind is simply modeled using the Prandtl's mixing length hypothesis [3, 6],

$$
\frac{d U}{d y}=\frac{\left[\left(S^{*}-s\right) \sigma\right]^{1 / 2}}{\kappa\left(y+y_{0}\right)},
$$

where $\kappa=0.41$ is von Karman's constant, $l=y+y_{0}$ is the mixing length, and $y_{0}$ is the aerodynamical roughness length. For turbulent flows over a rough bed, $y_{0}=d / 30$. The dimensionless friction velocity $u^{*}$ is related to $S^{*}$ by $u^{*}=\sqrt{\sigma S^{*}}$.

\section{TRANSPORT OVER A RIGID BED}

\section{A. Boundary conditions at the bed}

We first treat the case of saltation transport on a rigid, bumpy bed. The boundary conditions at the bed result from the consideration of the rebound from the bed.

Numerical simulations of a single sphere shot onto a bumpy surface indicate [20, 23] that the average magnitude, $\xi^{+}$, and average vertical component, $\xi_{y}^{+}$, of the rebound velocity of an incident particle are given in terms of their incident values and the angle $\theta$ between the bed and the incident trajectory by

$$
\xi^{+}(0)=e(\theta) \xi^{-}(0)
$$

and

$$
\xi_{y}^{+}(0)=-e_{y}(\theta) \xi_{y}^{-}(0),
$$

where $e=a-b \sin \theta$ and $e_{y}=a_{y} / \sin \theta-b_{y}$, in which $a, b, a_{y}$ and $b_{y}$ are numerical constants. The simulations show [23] that these constants depend upon the normal coefficient of restitution, $\varepsilon_{n}$, characterizing the dissipation in a binary collision. For $\varepsilon_{n}=0.8$, numerical simulations yield: $a=0.9, b=0.25, a_{y}=0.65$ and $b_{y}=0$ (see Fig. 2). The value chosen for $\varepsilon_{n}$ is close to to that determined experimentally with spherical glass bead [10]. A slight variation of the value of $\varepsilon_{n}$ marginally changes the values of the parameters $\left(a, b, a_{y}\right.$, and $\left.b_{y}\right)$ of the rebound law.

\section{B. Formulation}

We must phrase and solve a two-point boundary-value problem for the seven independent variables $\xi_{x}^{+}, \xi_{x}^{-}, \xi_{y}^{+}, \xi_{y}^{-}$, $x^{+}, x^{-}$and $U$ between $y=0$ and $y=H$. In addition, there are three unknown parameters to be determined: the height $H$ and length $L$ of the particle trajectory, and the vertical particle flux at the bed, $\phi_{0}$. 


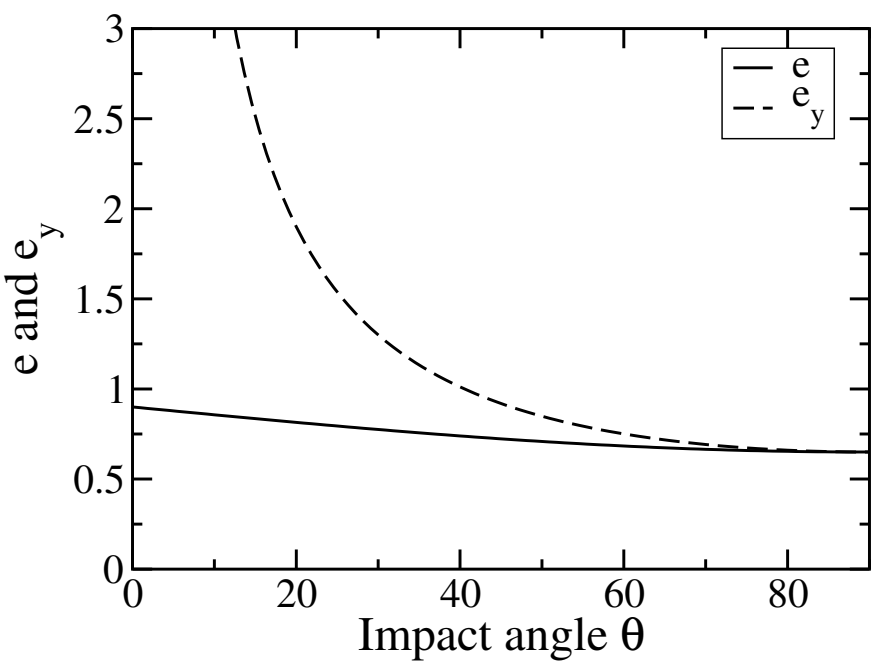

FIG. 2: Effective restitution coefficients $e$ and $e_{y}$ that characterize the collision of a single particle with a rigid, bumpy bed.

Kinematic relations provide seven conditions:

$$
\begin{aligned}
x^{+}(0) & =0, \\
x^{-}(0) & =L, \\
x^{+}(H) & =x^{-}(H), \\
\xi_{x}^{+}(H) & =\xi_{x}^{-}(H), \\
\xi_{y}^{+}(H) & =0, \\
\xi_{y}^{-}(H) & =0,
\end{aligned}
$$

and

$$
U(0)=0
$$

while the collision relations at the bed give two additional conditions. We have, then, only nine boundary conditions, so one parameter is left free. To close the problem on the rigid bed, we must prescribe the amount of particles in the flow through the particle hold-up, $M \equiv \int_{0}^{H} c(y) d y$, where $c=c^{+}+c^{-}$.

The two-point boundary-value problem for $\xi_{x}^{+}, \xi_{x}^{-}, \xi_{y}^{+}, \xi_{y}^{-}, x^{+}, x^{-}$and $U$ is solved using the built-in Matlab function bvp4c. Because there are nine boundary conditions and seven independent variables, the unknown parameters $H$ and $L$ are determined as part of the solution. The solution of the problem depends on two independent control parameters: the particle hold-up, $M$, and the Shields parameter, $S^{*}$.

\section{Results for a rigid bed}

As in the experiments of Ho et al. [11], we consider sand grains in air; for these, $d=230 \mu m, \rho^{s} / \rho^{f}=2,200$, $R=0.73$ and $\mu^{f} / \rho^{f}=0.15$.

In Fig. 3, we plot the particle trajectories at a fixed Shields parameter $S^{*}=0.06$ for increasing particle hold-ups. For small hold-ups, the height and length of the trajectories remain almost unchanged with increasing hold-up. However, above a critical particle hold-up, the increasing number of saltating particles begins to reduce the strength of the wind, resulting in a decrease in the height and length of the particle trajectory. This crossover is expected to occur when the particle shear stress $s$ becomes comparable with the air shear stress $S$ within the saltation layer. As shown in Appendix A, the particle shear stress at the bed is, in a first order approximation, linearly related to the particle hold-up:

$$
s_{0} \approx M / 2 \alpha
$$

where $\alpha$ is an effective restitutive coefficient that characterizes how much of the horizontal momentum lost by the particle at the impact is converted into vertical momentum (see details in Appendix A). In a steady state, the sum of 

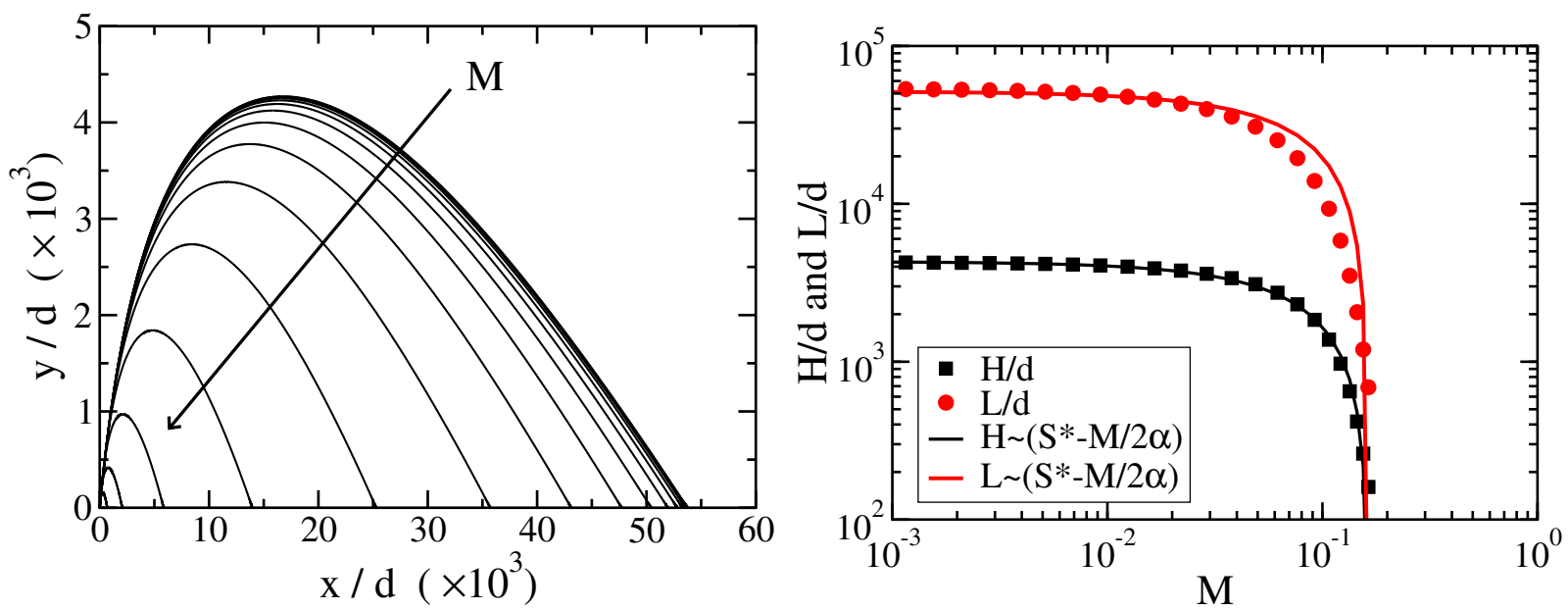

FIG. 3: (a) Steady particle trajectories at fixed Shields parameter $S^{*}=0.06$ for particle hold-ups ranging from 0.001 to 0.16 . (b) Variation of the trajectory height and length as a function of the particle hold-up at fixed Shields parameter, $S^{*}=0.06$. Solid lines represent fits using the function $S^{*}-M / 2 \alpha$ derived from the analytical calculation. The best fit gives $\alpha=1.4$.

the shear stresses in the particles and air is uniform through the height and equal to the Shields number $\left(s+S=S^{*}\right)$. Thus, we expect that the height and length of the particle trajectory begin to decrease when $s_{0}$ is of the order of the Shields parameter; that is, when $M \sim S^{*}$. The analytical calculation (see Appendix A) shows that the trajectory height $H$ should scale linearly with the air shear stress at the bed $S_{0}: H \approx S_{0}-S_{c}^{*} \approx S^{*}-S_{c}^{*}-s_{0} \approx S^{*}-S_{c}^{*}-M / 2 \alpha$, in which $S_{c}^{*}$ is the Shields parameter at which saltation ceases. The results of the periodic simulation are in good agreement with this simple scaling for $H$ and $L$ (see Fig. $3 \mathrm{~b}$ ).

In Figs. 4a and 4b, we plot the evolution of the air velocity profile for increasing particle hold-up at a fixed Shields parameter $S^{*}=0.06$ and the corresponding particle velocity profile. The air velocity within the saltation layer (i.e., for $y<H$ ) follows a logarithm law, which is well approximated by

$$
U(y) \approx \frac{\sqrt{\sigma S_{0}}}{\kappa} \ln \frac{y}{y_{0}},
$$

where $S_{0}$ is the air shear stress at the bed. As mentioned above, $S_{0}\left(\approx S^{*}-S_{c}^{*}-M / 2 \alpha\right)$ decreases with increasing particle hold-up. Above the saltation layer (i.e., $y>H)$, the air velocity is still logarithmic but with a different slope set by the Shields parameter. The kink in the air velocity profile indicates the crossover between the saltation layer and the free stream. In contrast, the particle velocity profile is almost flat $\left(u(y) \approx u(0) \equiv u_{0}\right)$. As expected, as the hold-up increases, the strength of flow is progressively reduced, resulting in a decrease of the particle velocity (see Fig. 5a). Assuming that the particle velocity at the bed is proportional to the air speed within the saltation layer, we obtain a simple scaling for $u_{0}$ as a function of the Shields number and the particle hold-up:

$$
u_{0} \approx \gamma \sqrt{\sigma\left(S_{0}-S_{c}^{*}\right)} \approx \gamma \sqrt{\sigma}\left(S^{*}-S_{c}^{*}-M / 2 \alpha\right)^{1 / 2}
$$

where $\gamma$ is a constant. The periodic simulation corroborates this scaling (the solid line in Fig. 5a).

Interestingly, the particle concentration profile (not shown here) is almost uniform through the height $(c(y) \approx$ $\left.c(0) \equiv c_{0}\right)$ and increases with increasing particle hold-up (see Fig. 5b). An approximate analytical calculation (see Eq. A15 in Appendix A) shows that the particle concentration at the bed can be approximated by $c_{0} \sim M / 2 H \approx$ $M / \sigma\left(S^{*}-S_{c}^{*}-M / 2 \alpha\right)$. This agrees well with the results of perioidic simulation (the solid line in Fig. $5 \mathrm{~b}$ ).

Another interesting quantity is the total particle flow rate, $Q \equiv \int_{0}^{H} c(y) u(y) d y$. In Fig. 6, we plot the evolution of $Q$ as a function of the particle hold-up for a fixed Shields parameter. Here, $Q$ first increases with increasing hold-up, reaches a maximum and then decreases. This evolution can be easily explained considering that the particle flow rate can be approximated by $Q \approx M u_{0}$. Because at moderate hold-up, the particle velocity is almost unchanged with increasing hold-up, the particle flow rate first increases linearly with increasing hold-up, $Q \sim M$. At larger hold-ups, the particle velocity decreases drastically with increasing hold-up, resulting in a decrease of the particle flow rate. Taking advantage of the approximate scaling proposed for the particle velocity $u_{0}$, we obtain a simple expression for the particle flux:

$$
Q \sim M\left(S^{*}-S_{c}-M / 2 \alpha\right)^{1 / 2}
$$



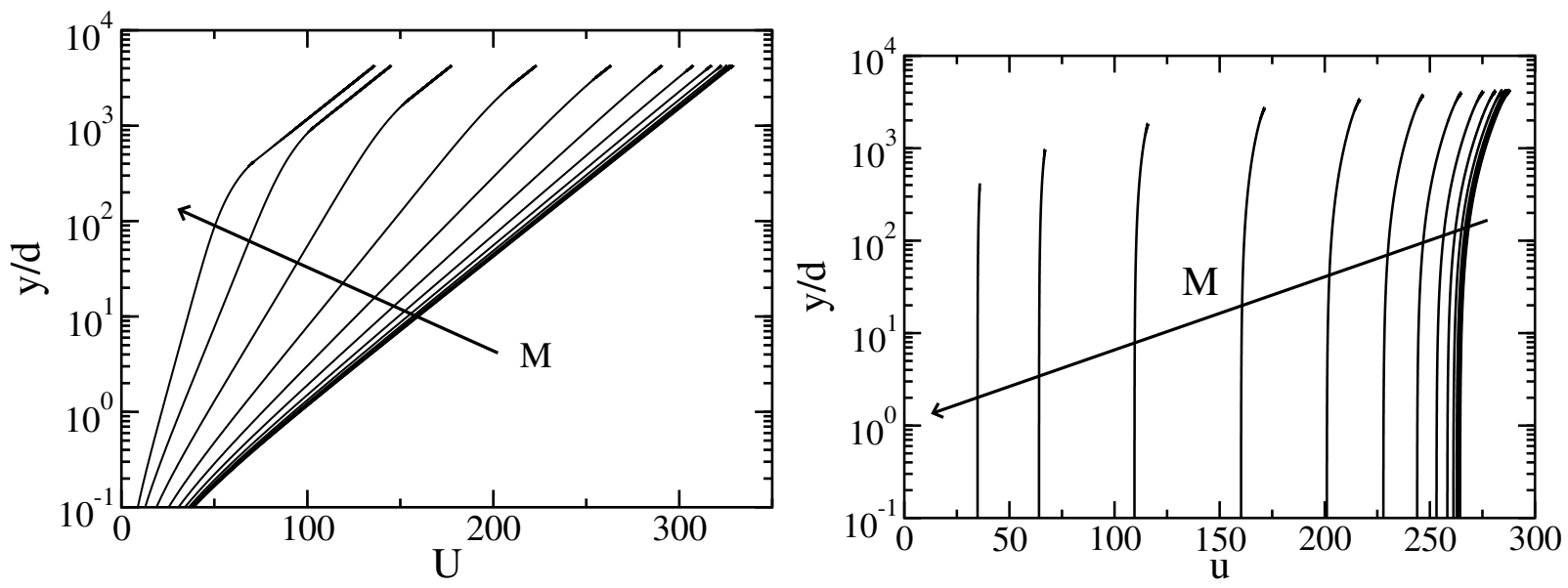

FIG. 4: Air (a) and particle (b) velocity profiles for particle hold-ups ranging from 0.001 to 0.16 at a fixed Shields parameter, $S^{*}=0.06$. The kink in the air velocity profile indicates the crossover between the saltation layer and the free stream.
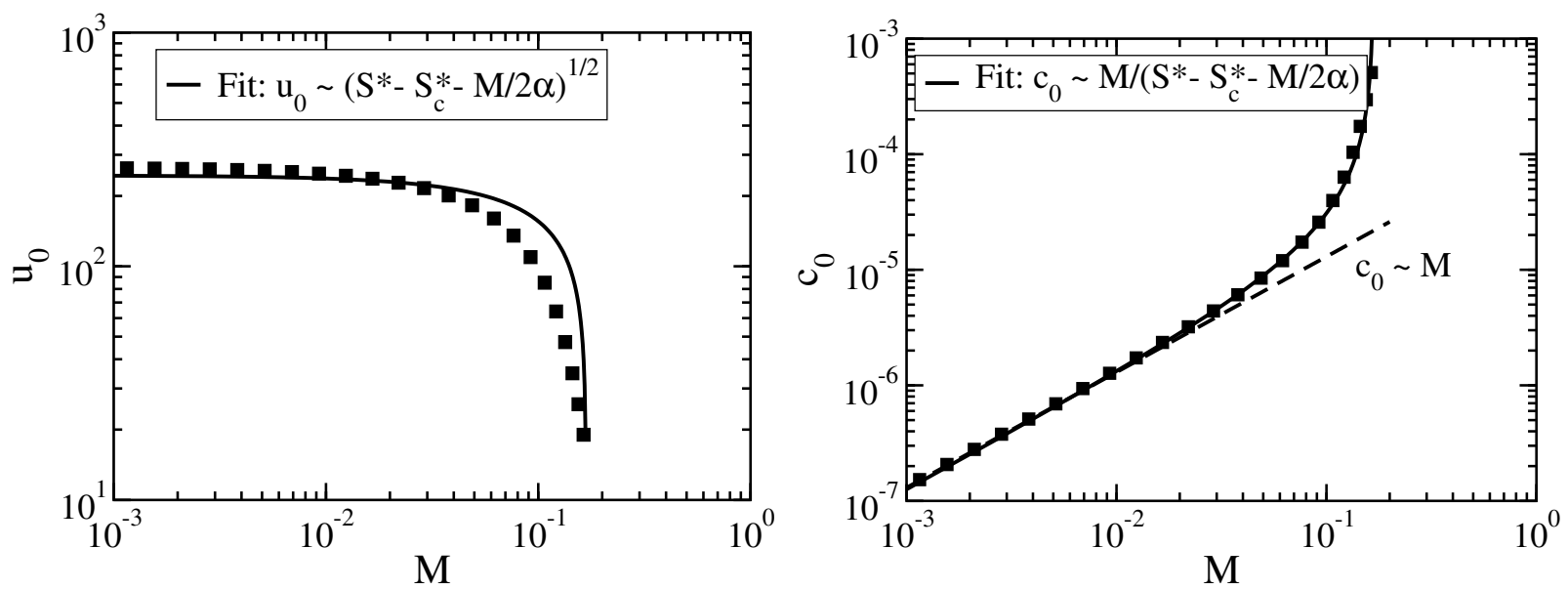

FIG. 5: Particle velocity (a) and particle concentration (b) at the bed as functions of the particle hold-up at a fixed Shields parameter, $S^{*}=0.06$. Solid lines are fits using expressions derived from analytical calculation. Best fits give $\alpha \approx 1.3$.

As a consequence, there exists a particle hold-up, $M_{\max }$ at which the particle flow rate is a maximum, $Q_{\max }$. Also, there exist two different particle hold-ups at a given particle flow rate: one at a small mass hold-up, with fast particles; and the other at larger mass hold-up, with slow particles. We expect, however, that the latter is not stable.

The above features of saltation transport remain qualitatively unchanged as the Shields parameter is varied. There is only a quantitative change. For example, the particle flow rate increases with increasing Shields parameter and still exhibits a peak at a maximum particle hold-up, $M_{\max }$, (see Fig. 6). The maximum hold-up is found to scale linearly with the Shield parameter, $M_{\max } \propto S^{*}-S_{c}^{*}\left(S_{c}^{*} \approx 0.002\right.$, as, for example, in Fig. 7a). This scaling is readily obtained upon taking the derivative of Eq. 26, which yields

$$
M_{\max } \approx 4 \alpha\left(S^{*}-S_{c}^{*}\right) / 3
$$

The height and length of the particle trajectory at the maximum are also found to scale linearly with $S^{*}-S_{c}^{*}($ see Fig. 7b).

In contrast, the particle velocity at the maximum scales with the square root of the Shields parameter, $u_{0 \text { max }} \propto$ $\sqrt{S^{*}-S_{c}^{*}}$, (see Fig. 8a). This scaling can be obtained by employing Eq. 27 in Eq. 25.

A direct consequence of these results is that the maximum particle flow rate is expected to scale as $Q_{\max } \propto$ $\left(S^{*}-S_{c}^{*}\right)^{3 / 2}$ (see Fig. 8b). This prediction is in agreement with the experimental scaling found by Ho et al. [11] for saltation transport over a rigid, bumpy bed. Furthermore, the agreement is also quantitative (see Fig. 9). 


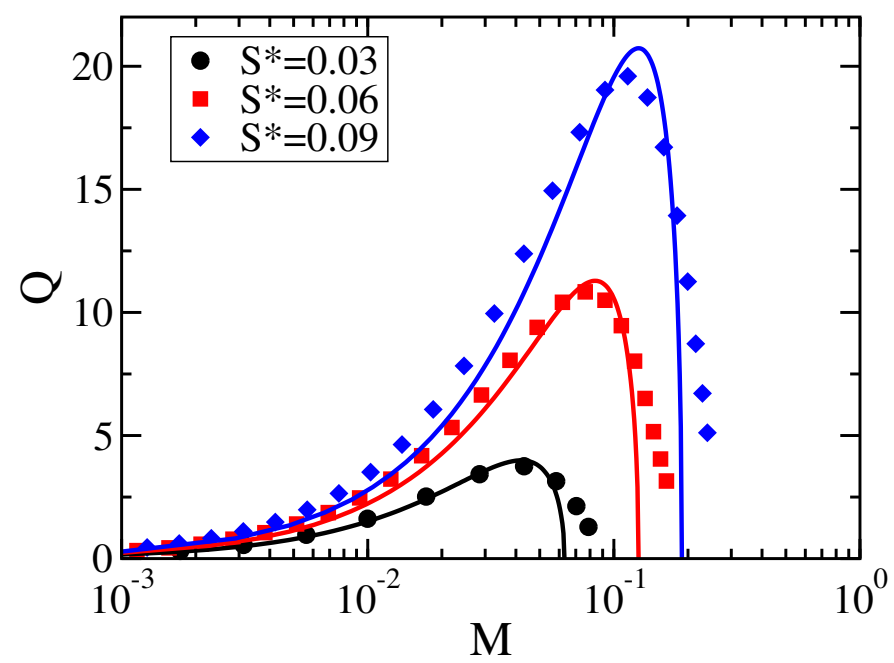

FIG. 6: Particle flow rate $Q$ as a function of the particle hold-up for Shields parameters $S^{*}=0.03,0.06,0.09$. The solid lines are fits using the approximation $Q \approx M\left(S^{*}-M / 2 \alpha\right)^{1 / 2}$. The best fits give $\alpha=1.2$.
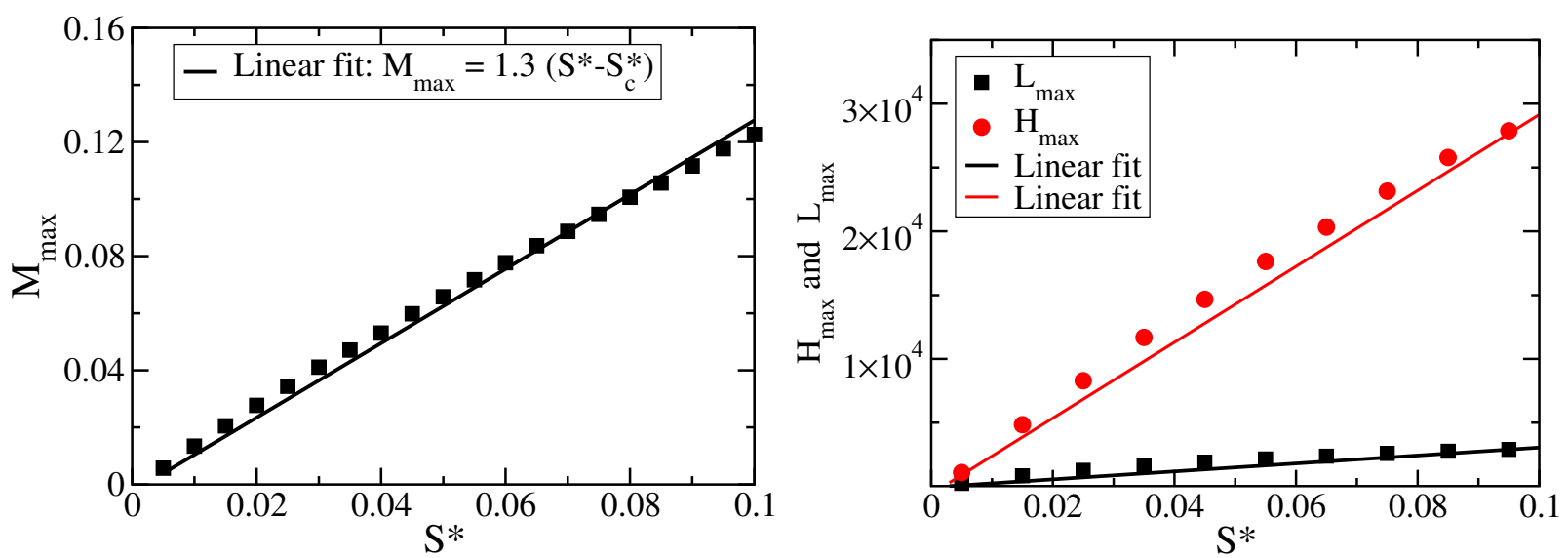

FIG. 7: (a) Particle hold-up at the maximum flux as a function of the Shields parameter: Periodic simulation (symbols) and linear fit (solid line). (b) Trajectory height and length at the maximum particle flow rate, $H_{\max }$ and $L_{\max }$, as a function of the Shields parameter: Periodic simulation (symbols) and linear fit.

\section{TRANSPORT OVER AN ERODIBLE BED}

The major change for saltation transport over an erodible bed is in the boundary conditions at the bed. Over a rigid bed, the impact velocity in a periodic trajectory is unconstrained; while over an erodible bed, it must have a value that produces a single rebounding particle.

\section{A. Erodible boundary conditions}

The nature of the rebound remains qualitatively unchanged and can be described by Eqs. 14 and 15 . The only difference is quantitative: the dissipation is increased on an erodible bed. As a consequence, the parameters $a, b, a_{y}$ and $b_{y}$ take different values. Experiments [21] on single spheres shot into a bed of like spheres permit the determination of theses values: $a=0.87, b=0.72, a_{y}=0.30$ and $b_{y}=0.15$. Numerical simulations [20] show that these values are independent of the diameter of the spheres, but depend upon their coefficients of restitution.

The other aspect of the collision process is the ejection of particles from the bed. Experiments [17, 21] show the 

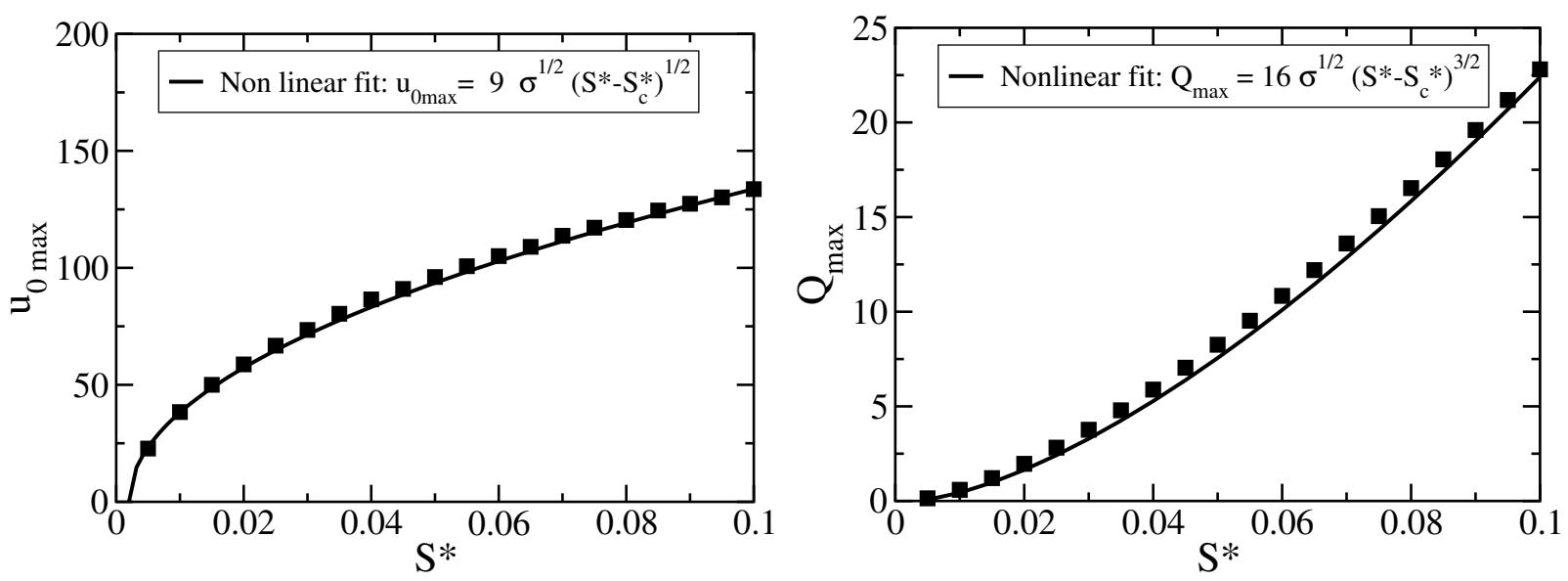

FIG. 8: (a) Particle velocity at the bed at the maximum particle flow rate, $u_{0 \max }$ as a function of the Shields parameter: Periodic simulation (symbols) and fit using a square root function (solid line). (b) Maximum particle flow rate, $Q_{\max }$, as a function of the Shields parameter: Periodic simulation (symbols) and fit using a power function with $3 / 2$ exponent (solid line).

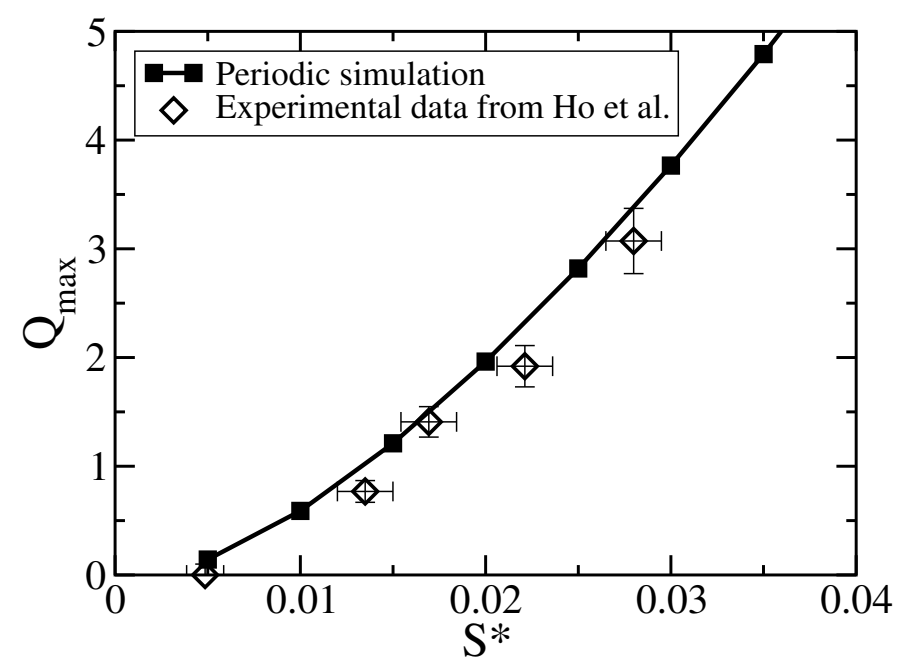

FIG. 9: Comparison between experiments and simulation: (solid line) numerical results of the periodic simulation and (symbols) experimental data from Ho et al. [11].

the number $N$ of ejected particles, including the rebound, is given by:

$$
N(\xi)= \begin{cases}1+N_{0}\left(1-e^{2}\right)\left(\xi / \xi_{c}-1\right) & \text { if } \xi>\xi_{c} \\ 1 & \text { if } 1 \leq \xi \leq \xi_{c} \\ 0 & \text { if } \xi \leq 1\end{cases}
$$

where $\xi_{c}$ is the threshold velocity below which there is no ejection. The measurements indicate that $N_{0}=13$ and $\xi_{c}=40$. This relation indicates that the number of ejected particles is the product of a function $1-e^{2}(\theta)$ that depends only on the impact angle and a linear function of the impact velocity above a threshold $\xi_{c}$. If the velocity of the impacting particle is less than unity (i.e., $\sqrt{g d}$ in physical units), the impacting particle is captured by the bed.

In a steady state, the sum of the upward and downward vertical particle flux through the bed surface should be zero $\left(\phi_{0}^{+}+\phi_{0}^{-}=0\right)$, so $N=1$. In other words, a particle impact on the bed results on average in a single rebounding particle. Used with the collision relation (28), the latter condition requires the impact velocity $\xi^{-}(0)$ of the saltating particle to lie between 1 and $\xi_{c}$. For the average periodic trajectory that we consider, we take the average, $\xi_{c} / 2$, of these limiting values to be the impact velocity.

Zero vertical particle flux provides one more boundary condition than in the rigid case. We have, then, ten boundary conditions for seven independent variables. Consequently, the three unknown parameters $H, L$, and $\phi_{0}$ are determined 
as part of the solution. The particle hold-up $M$ is no longer a free parameter, but is determined, because of the balance between erosion and deposition.

\section{B. Results for an erodible bed}

We consider again sand grains of diameter $d=230 \mu \mathrm{m}$ and relative density $\rho^{s} / \rho^{f}=2,200$.

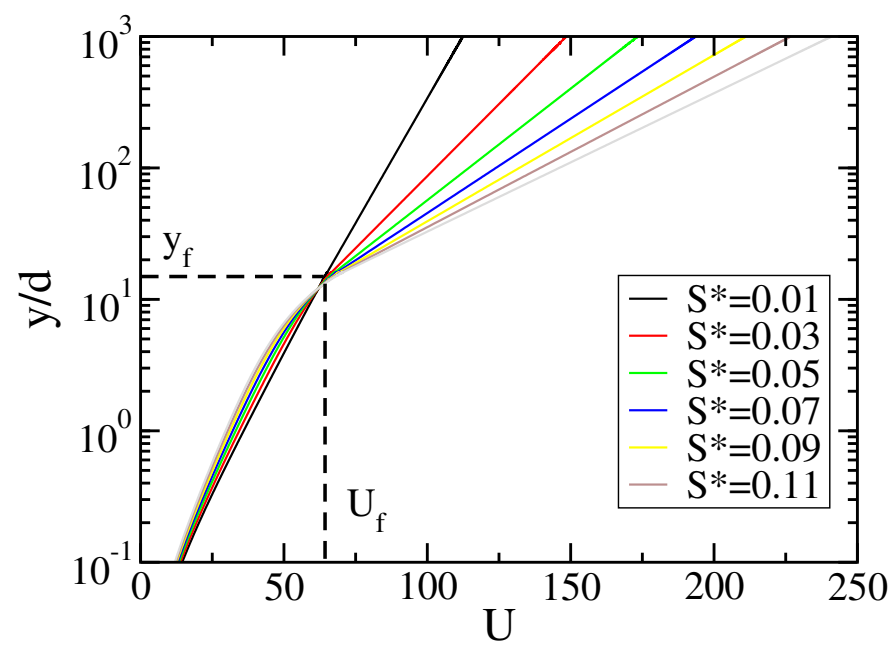

FIG. 10: Air velocity profiles for Shields parameters ranging from 0.01 to 0.1 . The profiles intersect at a focus point with coordinates $\left(y_{f}, U_{f}\right): y_{f}=16$ and $U_{f}=62$.

The numerical solution of the system of equations provides the air velocity profiles in the steady state. Fig. 10 show the profiles for various Shields parameters. As first evidenced by Bagnold [1], the air velocity profiles cross at a focus point. Below the focus, the air speed is almost independent of the strength of the wind as found by more elaborate models [2-6]. The height of the focus, $y_{f}$, coincides with the height of the periodic particle trajectory: $y_{f} \approx H \approx 16$. The value found for the focus height is underestimated in comparison with experimental values from the literature. (For example, wind-tunnel experiments [3, 24] indicate that $y_{f} \approx 30$ for particle diameter $d=230 \mu m$ ). This underestimation is probably caused by the fact that our model considers a single, average particle trajectory.

In Fig. 11a, we plot steady periodic trajectories for various Shields parameters. The particle trajectory is found to be independent of the wind strength. This result is not surprising, because the particle velocity at the bed is fixed by the splash process. The height and length are, thus, independent of the Shields parameter, in agreement with recent experimental measurements [25] (see Fig. 11b). The agreement between the periodic simulation and the experimental results from Ho et al. [25] is also quantitative.

The only quantity that varies as the wind strength increases is the particle concentration. The particle concentration adjusts, so that the air velocity is reduced, in order to transport the particles at the velocity prescribed by the splash. The particle concentration is rather uniform through the height, $\left(c(y) \approx c_{0}\right)$, as in the rigid case, and increases linearly with the Shields parameter: $c_{0} \propto S^{*}-S_{c}^{*}$ (see Fig. 12a). A consequence of this is that the particle hold-up $M \approx c_{0} H$ is also a linear function of the Shields parameter. This is the same scaling as that obtained for transport on a rigid bed at the maximum particle flux and can be derived from simple physical arguments, as shown in Appendix B. The linear scaling of the particle concentration with the Shields parameter is in agreement with experiments [3], but the limitation of the simple model is that it is not able to reproduce the exponential decrease of the particle concentration with height. This weakness is inherent to our consideration of the single, average particle trajectory, and can be redressed by considering a distribution of particle trajectories, as is done in more elaborate models [3, 4, 6].

As expected, the particle velocity at the bed $u_{0}$ is independent of the wind strength (see Fig. 12b). This contrasts with the rigid case, in which it scaled as the square root of the Shields parameter, and had an important influence on the scaling of the particle flow rate.

In Fig. 13, we plot the particle flow rate as a function of the Shields parameter. In contrast with transport over a rigid bed, where $Q \sim S^{* 3 / 2}$ for the maximum particle flux, the particle flow rate over an erodible bed varies linearly with the Shields parameter. This is a direct consequence of the independence of the particle velocity on the wind strength, $Q \approx u_{0} c_{0} H \propto S^{*}-S_{c}^{*}$. This linear scaling has also been seen experimentally [3, 11]. The prediction of the 

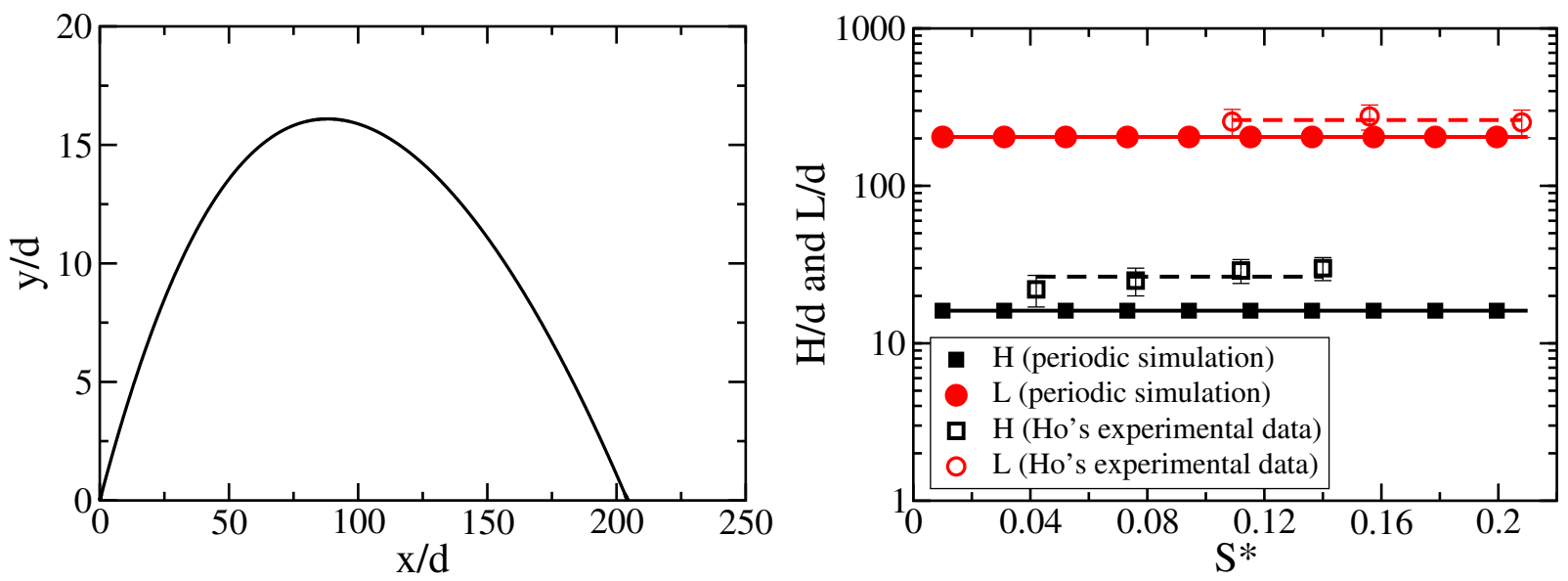

FIG. 11: (a) Steady trajectories for Shields parameters $S^{*}=0.01,0.05,0.1,0.15$. (b) Trajectory height and length versus the Shields parameter. The dashed lines are the experimental estimations from Ho et al. [25].
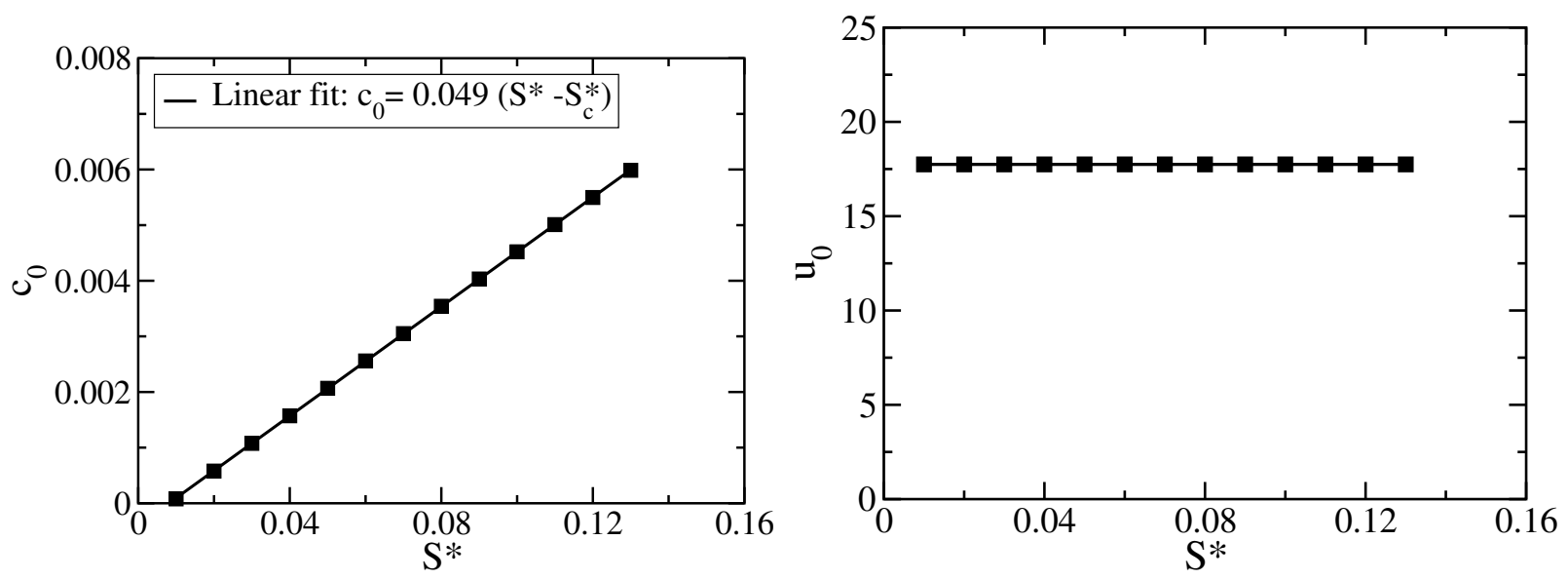

FIG. 12: (a) Particle concentration the bed as a function of the Shields parameter: periodic simulation (symbols) and linear fit (solid line). b) Particle velocity at the bed as a function of the Shields parameter.

model provides quantitative agreement with the experimental data [11, 24] (see Fig. 13). The numerical results of the periodic simulation indicate that the critical Shields parameter at which saltation ceases on a erodible bed is higher than in the rigid case $\left(S_{c}^{*} \approx 0.008\right.$ for an erodible bed), as found in the experiments [11, 24].

\section{CONCLUSION}

We have phrased and solved a two-point boundary-value problem for periodic particle trajectories in steady, uniform turbulent shearing flows above horizontal beds. We considered two types of beds: a bumpy, rigid bed; and an erodible, particle bed. Particles collide and rebound from a rigid bed without constraint; while the impact velocity in a collision with an erodible bed must result in a single product particle. Rigid beds support a range of particle flow rates up to a maximum, $Q_{\max }$, given the strength of the turbulent shear, the Shields parameter, $S *$. Erodible beds support a single particle flow rate at a given Shields parameter.

For rigid beds, $Q_{\max }$ is proportional to $\left(S^{*}-S_{c}^{*}\right)^{3 / 2}$. For erodible beds, $Q$ is proportional to $S^{*}-S_{c}^{*}$. Such relations are confirmed by experiment, as are the properties of the height and length of the trajectories and the average velocity profiles of the wind and particles measured in the simulations and predicted analytically. However, not all features measured in experiments and simulations are captured: the predicted concentration is constant, rather than decaying exponentially with distance from the bed. In any case, the combination of the conceptual simplicity and the predictive powers of the periodic trajectories make them a powerful tool for the understanding of 


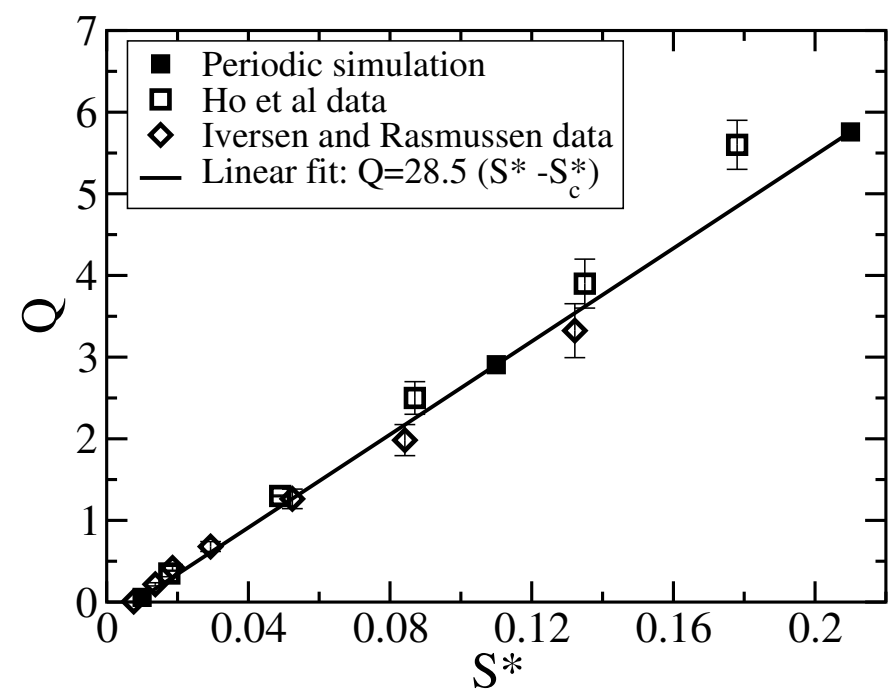

FIG. 13: Particle flux as a function of the Shields parameter derived from the model (solid line). The model prediction is compared with the experimental data of Ho et al. [11] (open square) and of Iversen and Rasmussen [24] (open diamond) carried out with grain size $d=230 \mu m$ and $d=242 \mu m$, respectively.

Aeolian sand transport.

Acknowledgment - This research was supported in part by the National Science Foundation under Grant No. NSF PHY11-25915 to the Kavli Institute of Theoretical Physics. J. T. Jenkins is grateful to the University of Rennes 1 for the support of research visits.

\section{Appendix A: Analytical derivation of scalings for transport over a rigid bed}

We provide here the analytical derivation of scalings obtained for transport over a rigid bed. To do so, we make a drastic simplification and assume that the vertical air drag acting on the particle is negligible. This assumption is not justified - numerical calculations indicate that vertical drag is significant - but it makes the analytical calculation tractable.

We first derive the relation between the particle hold-up, $M$, and the particle concentration at the bed, $c_{0}$. With the assumption regarding the vertical air drag, the upward and downward vertical particle velocities are equal and opposite (i.e., $\xi_{y}^{+}=-\xi_{y}^{-}$) and the upward and downward particle concentration are equal (i.e., $c^{+}=c^{-}$). In addition, the upward vertical particle velocity is simply related to its vertical position:

$$
\xi_{y}^{+2}(y)=2(H-y) .
$$

Using the above relation together with Eq. 11, we obtain an expression for the upward particle concentration as a function of the vertical position:

$$
c^{+}(y)=\frac{\phi_{0}}{\xi_{y}^{+}(y)}=\frac{c_{0}^{+} \sqrt{2 H}}{\sqrt{2(H-y)}} .
$$

Integrating the concentration through the height, we determine the expression for the particle hold-up as a function of the particle concentration $c_{0}$ at the bed $\left(c_{0}=c_{0}^{+}+c_{0}^{-}=2 c_{0}^{+}\right)$and the trajectory height $H$ :

$$
M=\int_{0}^{H} d y\left[c^{+}(y)+c^{-}(y)\right]=4 c_{0}^{+} H=2 c_{0} H .
$$

We now proceed to the derivation of the particle flux. Using the fact that the particle velocity is rather uniform through the height, the particle flux $Q$ can be well approximated by

$$
Q=\int_{0}^{H} d y c(y) u(y) \approx M u_{0},
$$


where $u_{0}$ is the average particle velocity at the bed [i.e., $u_{0}=\left(\xi_{x 0}^{+}+\xi_{x 0}^{-}\right) / 2$ ].

The particle velocity is governed by the strength of the air flow within the saltation layer. We expect, in particular, that the particle velocity at the bed should scale as the square root of the air shear stress at the bed, $S_{0}$. This is indeed confirmed by the periodic simulation (see Fig. 14):

$$
u_{0} \approx \gamma \frac{\sqrt{\sigma\left(S_{0}-S_{c}^{*}\right)}}{\kappa}
$$

where $\gamma$ is a proportionality constant.

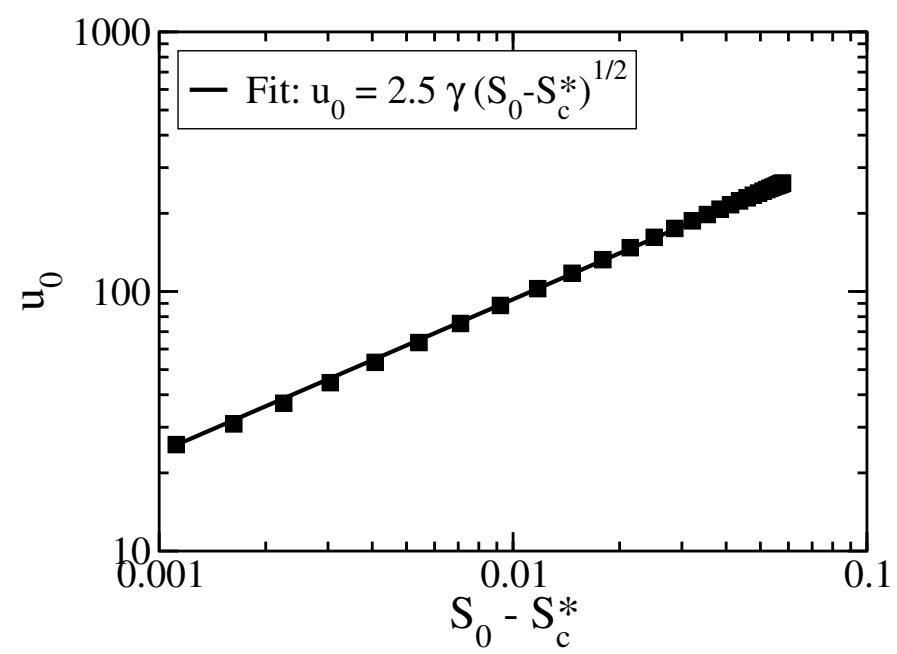

FIG. 14: Particle velocity at the bed as a function of the air shear stress at the bed for increasing particle hold-ups $(0.001<$ $M<0.12$ ) at a fixed Shields parameter, $S^{*}=0.06$ : Periodic simulation (symbols) and fit using a square root function (solid line). The best fit gives $S_{c}^{*} \approx 0.002$ and $\gamma \approx 6$.

It remains to relate the air shear stress at the bed to the distant air shear stress $S^{*}$ and the particle hold-up. In a steady state, the sum of the particle and air shear stress (respectively, $s$ and $S$ ) is constant through the height and equal to the distant shear stress $\left(s+S=S^{*}\right)$. Thus, at the bed, we have

$$
S_{0}=S^{*}-s_{0}
$$

The particle shear stress at the bed is given by

$$
s_{0}=c_{0}^{+} \xi_{y 0}^{+}\left(\xi_{0 x}^{-}-\xi_{0 x}^{+}\right) .
$$

Introducing an effective restitutive coefficient, $\alpha$, to characterize how much of the horizontal momentum lost by the particle at the impact is converted into vertical momentum,

$$
\alpha=\frac{\xi_{y 0}^{+}}{\xi_{0 x}^{-}-\xi_{0 x}^{+}},
$$

we have

$$
s_{0}=\frac{c_{0}^{+} \xi_{y 0}^{+2}}{\alpha}=\frac{2 c_{0}^{+} H}{\alpha}=\frac{M}{2 \alpha},
$$

where we used the fact that $\xi_{y 0}^{+2}=2 H$ and $M=4 c_{0}^{+} H$ (c.f., Eqs. A1 and A3). Note that $\alpha$ can be expressed as a function of the restitutive coefficients $e$ and $e_{y}$ introduced previously:

$$
\alpha=e_{y} \tan \theta /(1+\beta)
$$

where $\beta=\xi_{0 x}^{+} / \xi_{0 x}^{-}=\left[e^{2}+\left(e^{2}-e_{y}^{2}\right) \tan \theta\right]^{1 / 2}$. Eq. A9 provides us with an approximate expression for the particle shear stress at the bed. The periodic simulation indicates that Eq. A9 provides an approximation that is within $10 \%$ (see Fig. 15). 


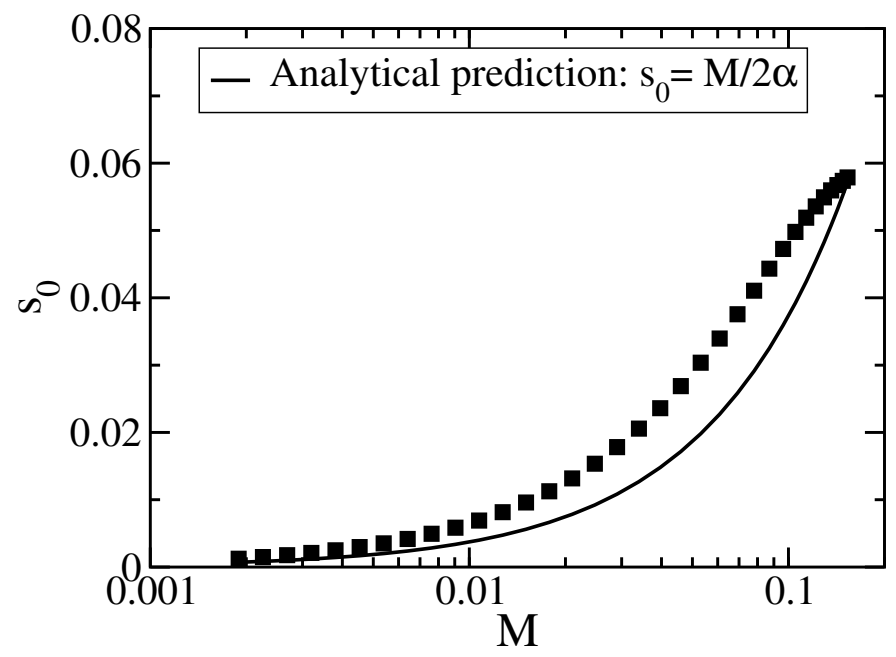

FIG. 15: Particle shear at the bed as a function of particle hold-up at a fixed Shields parameter, $S^{*}=0.06$ : Periodic simulation (symbols) and linear approximation of the form $M / 2 \alpha$ with $\alpha=1.4$ (solid line). The value of $\alpha$ is taken from the numerical evaluation of Eq. A8.

Using Eqs. A6 and A9, we finally determine an expression for the air shear stress at the bed as a function of the particle hold-up:

$$
S_{0}=S^{*}-M / 2 \alpha
$$

Employing this relation in Eq. A5, we obtain an approximate expression for the particle velocity

$$
u_{0} \approx \frac{\gamma \sqrt{\sigma}}{\kappa} \sqrt{S^{*}-S_{c}^{*}-M / 2 \alpha}
$$

which, used in Eq. A4, yields:

$$
Q=\frac{\gamma \sqrt{\sigma}}{\kappa} M \sqrt{S^{*}-S_{c}^{*}-M / 2 \alpha}
$$

This is the expression sought for the particle flux.

We can also deduce an expression for the trajectory height:

$$
\begin{aligned}
H & =\frac{\xi_{y 0}^{+2}}{2}=\frac{2 \alpha^{2}(1-\beta)^{2}}{(1+\beta)^{2}} u_{0}^{2} \\
& \approx \frac{2 \alpha^{2}(1-\beta)^{2} \gamma^{2} \sigma}{\kappa^{2}(1+\beta)^{2}}\left(S^{*}-S_{c}^{*}-M / 2 \alpha\right),
\end{aligned}
$$

and for the particle concentration at the bed $c_{0}$,

$$
c_{0}=\frac{M}{2 H} \approx \frac{\kappa^{2}(1+\beta)^{2}}{4 \alpha^{2}(1-\beta)^{2} \gamma^{2} \sigma} \frac{M}{\left(S^{*}-S_{c}^{*}-M / 2 \alpha\right)} .
$$

The maximum particle hold-up is easily obtained from Eq. A13:

$$
M_{\max }=\frac{4 \alpha}{3}\left(S^{*}-S_{c}^{*}\right)
$$

and $Q_{\max }$ is given by

$$
Q_{\max }=\frac{4 \alpha \gamma \sqrt{\sigma}}{3 \sqrt{3} \kappa}\left(S^{*}-S_{c}^{*}\right)^{3 / 2} .
$$


The parameters $\alpha, \beta$ and $\gamma$ are actually not constant, but depend on the exact shape of the particle trajectory. Periodic simulations indicate that for the range of particle hold-up and Shields parameter investigated so far (i.e., $0.01<S^{*}<0.1$ and $0.001<M<0.1$ ), $\alpha$ and $\beta$ vary, respectively, from 1.35 to 1.45 and 0.45 to 0.55 , whereas $\gamma$ ranges from 5 to 7 . Taking average values for theses parameters $(\alpha=1.4, \beta=0.5$ and $\gamma=6)$, we obtain

$$
M_{\max } \approx 1.8\left(S^{*}-S_{c}^{*}\right)
$$

and

$$
Q_{\max } \approx 17 \sqrt{\sigma}\left(S^{*}-S_{c}^{*}\right)^{3 / 2}
$$

which provide good estimates of the numerical prediction. Best fits to the numerical data give, respectively, $M_{\text {max }} \approx$ $1.3\left(S^{*}-S_{c}\right)$ and $Q_{\max } \approx 16 \sqrt{\sigma}\left(S^{*}-S_{c}^{*}\right)^{3 / 2}$, with $S_{c}=0.002$ (see Figs. 7a and 8b).

Finally, we give estimates of the particle velocity and the trajectory height at the maximum particle flux. Using Eq. A18 in Eqs. A12 and $A 14$ yields:

$$
u_{0 \max } \approx \frac{\gamma \sqrt{\sigma}}{\sqrt{3} \kappa}\left(S^{*}-S_{c}^{*}\right)^{1 / 2} \approx 9 \sqrt{\sigma}\left(S^{*}-S_{c}^{*}\right)^{1 / 2}
$$

and

$$
\begin{aligned}
H_{\max } & \approx \frac{2 \alpha^{2}(1-\beta)^{2} \gamma^{2} \sigma}{3 \kappa^{2}(1+\beta)^{2}}\left(S^{*}-S_{c}^{*}\right) \\
& \approx 20 \sigma\left(S^{*}-S_{c}^{*}\right) .
\end{aligned}
$$

The agreement with the periodic simulation is excellent for the particle velocity. Indeed, the best fit to the numerical data gives $u_{0 \max } \approx 9 \sqrt{\sigma}\left(S^{*}-S_{c}^{*}\right)^{1 / 2}$ (see Fig. 8a). In contrast, the estimation of $H_{\max }$ is $50 \%$ greater than the numerical result, for which the best fit gives $H_{\max } \approx 13.5 \sigma\left(S^{*}-S_{c}^{*}\right)$. This overestimation is not surprising, because we have neglected the vertical air drag acting on the particle in the analytical derivation.

\section{Appendix B: Analytical derivation of scalings for transport over an erodible bed}

We provide here the analytical derivation of scalings obtained for transport over an erodible bed. We assume again that the vertical air drag acting on the particle is negligible. In contrast with the rigid case, this assumption is quite reasonable for transport over an erodible because particles experience much smaller jumps.

As discussed in section IV, the particle hold-up is no longer a free parameter but is self-adjusted by the system. The additional constraint is provided by the splash, which sets the particle velocity at the bed in order to balance erosion and deposition. As seen in Sec. IV, this additional condition is

$$
\xi_{x 0}^{-} \approx \xi_{c} / 2
$$

where $\xi_{c}$ is the critical impacting velocity below which there is no ejected particles. Using the fact that $u_{0}=$ $(1+\beta) \xi_{x 0}^{+} / 2$, the average particle velocity at the bed, $u_{0}$, is

$$
u_{0}=\frac{(1+\beta)}{4} \xi_{c}
$$

The analytical expressions derived for transport over a rigid still apply in the present case, except that the particle hold-up, $M$, is not a free parameter. If we distinguish between the critical Shields parameter, at which saltation ceases for rigid and erodible beds, denoting these by $S_{R c}^{*}$ and $S_{E c}^{*}$, then $M$ is determined by equating the expression (A12) for $u_{0}$ with Eq. (B2):

$$
M=2 \alpha\left(S^{*}-S_{E c}^{*}\right),
$$

with $S_{E c}^{*}=S_{R c}^{*}+\kappa^{2}(1+\beta)^{2} \xi_{c}^{2} / 16 \gamma^{2} \sigma$. Using the fact that $Q \approx M u_{0}$, we find:

$$
Q=\frac{\alpha(1+\beta)}{2} \xi_{c}\left(S^{*}-S_{E c}^{*}\right)
$$

Periodic simulations indicate that the parameters $\alpha, \beta$ and $\gamma$ are fairly constant (i.e., independent of the Shields parameter $): \alpha \approx 0.65, \beta \approx 0.7$ and $\gamma \approx 2.3$. Using these values, we obtain $S_{E c} \approx 0.007$ and $Q \approx 30\left(S^{*}-S_{E c}^{*}\right)$ which are in fairly good agreement with the periodic simulations and the experimental data (see Fig. 13). 
We can also derive analytical expressions for the trajectory height and the particle concentration at the bed. Taking advantage of Eq. B3 together with Eqs. A14 and A15, we have

$$
H \approx \frac{\alpha^{2}(1-\beta)^{2}}{4} \xi_{c}^{2}
$$

and

$$
c_{0} \approx \frac{4}{\alpha(1-\beta)^{2} \xi_{c}^{2}}\left(S^{*}-S_{E c}^{*}\right)
$$

Using the values of $\alpha, \beta$ and $\gamma$ in these equations, we obtain $H \approx 15.3$ and $c_{0} \approx 0.043\left(S^{*}-S_{E c}^{*}\right)$. The agreement with periodic simulations is fairly good: $H \approx 16$ and $c_{0} \approx 0.05\left(S^{*}-S_{E c}^{*}\right)$ (see Figs. 11b and $12 \mathrm{a}$ ).

[1] R. A. Bagnold. The physics of blown sand and desert dunes. Methuen, New York, 1941.

[2] R. S. Anderson and P. K. Haff. Simulation of aeolian saltation. Science, 241:820-823, 1988.

[3] M. Creyssels, P. Dupont, A. Ould el Moctar, A. Valance, I. Cantat, J.T. Jenkins, J.M. Pasini, and K.R. Rasmussen. Saltating particles in a turbulent boundary layer : experiment and theory. Journal of Fluid Mechanics, 625:47-74, 2009.

[4] J.F. Kok and N.O. Rennó. A comprehensive numerical model of steady state saltation. Journal of Geophysical Research. Atmospheres, 114:D17204, 2009.

[5] M. V. Carneiro, T. Pähtz, and H. J. Herrmann. Jump at the onset of saltation. Phys. Rev. Lett., 107:098001, 2011.

[6] O. Duran, B. Andreotti, and P. Claudin. Numerical simulation of turbulent sediment transport from bed load to saltation. Phys. Fluids, 24:103306, 2012.

[7] M. V. Carneiro, N.A.M. Araujo, T. Pähtz, and H. J. Herrmann. Midair collisions enhance saltation. Phys. Rev. Lett., 111:058001, 2013.

[8] B. Andreotti. A two-species model of aeolian sand transport. Journal of Fluid Mechanics, 510:47-70, 2004.

[9] M. Lämmel, D. Rings, and K. Kroy. A two-species continuum model for aeolian sand transport. New J. Phys., 14:093037, 2012.

[10] A. Lorenz, C. Tuozzolo, and M.Y Louge. Measurements of impact properties of small, nearly spherical particles. Experimental Mechanics, 37:292-298, 1997.

[11] T. D. Ho, A. Valance, P. Dupont, and A. Ould El Moctar. Scaling laws in aeolian sand transport. Physical Review Letters, 106:094501, 2011.

[12] T. D. Ho, A. Valance, P. Dupont, and A. Ould El Moctar. Particle velocity distribution in saltation transport. Physical Review E, 85:052301, 2012.

[13] S. Mitha, M. Q. Tran, B. T. Werner, and P. K. Haff. The grain-bed impact process in aeolian saltation. Acta Mechanica, 63:267-278, 1986.

[14] J. Ungar and P. K. Haff. Steady state saltation in air. Sedimentology, 34:289-299, 1987.

[15] B. T. Werner and P. K. Haff. The impact process in aeolian saltation: two-dimensional simulations. Sedimentology, 35:189-196, 1988.

[16] B. B. Willetts and M. A. Rice. Collision of quartz grains with a sand bed: the influence of incident angle. Earth Surface Processes and Landforms, 14:719-730, 1989.

[17] B. T. Werner. A steady-state model of wind-blown sand transport. Journal of Geology, 98:1-17, 1990.

[18] F. Rioual, A. Valance, and D. Bideau. Experimental study of the collision process of a grain on a two-dimensional granular bed. Physical Review E., 62:2450-2459, 2000.

[19] M. A. Rice, B. B. Willetts, and I. K. McEwan. Observations of collisions of saltating grains with a granular bed from high-speed cine-film. Sedimentology, 43:21-31, 1996.

[20] L. Oger, M. Ammi, A. Valance, and D. Beladjine. Discrete element method to study the collision of one rapid sphere on 2d and 3d packings. The European Physical Journal E, 17:467-476, 2005.

[21] D. Beladjine, M. Ammi, A. Valance, and L. Oger. Collision process between an incident bead and a three-dimensional granular packing. Physical Review E., 75:061305, 2007.

[22] J. Dallavalle. Micrometrics. Pitman, New York, 1943.

[23] J. Crassous, D. Beladjine, and A. Valance. Impact of a projectile on a granular medium described by a collision model. Physical Review Letters, 99:248001, 2007.

[24] J.D. Iversen and K.R. Rasmussen. The effect of wind speed and bed slope on sand transport. Sedimentology, 46:723-731, 1999.

[25] T. D. Ho, A. Valance, P. Dupont, and A. Ould El Moctar. Aeolian sand transport: Length and height distributions of saltation trajectories. Aeolian Research, 12:65-74, 2014. 